\title{
Metamaterial with polarization and direction insensitive resonant transmission response mimicking electromagnetically induced transparency
}

\author{
N. Papasimakis, ${ }_{1}^{1}$ Y. H. Fu, ${ }^{1,2}$ V. A. Fedotov, ${ }^{1, a)}$ S. L. Prosvirnin, ${ }^{3}$ D. P. Tsai, ${ }^{2}$ and \\ N. I. Zheludev ${ }^{1, \mathrm{~b})}$ \\ ${ }^{1}$ Optoelectronics Research Centre, University of Southampton, Southampton SO17 1BJ, United Kingdom \\ ${ }^{2}$ Department of Physics, National Taiwan University, Taipei 10617, Taiwan \\ ${ }^{3}$ Institute of Radio Astronomy, National Academy of Sciences of Ukraine, Kharkov 61002, Ukraine
}

(Received 18 February 2009; accepted 24 April 2009; published online 26 May 2009)

\begin{abstract}
We report on a planar metamaterial, the resonant transmission frequency of which does not depend on the polarization and angle of incidence of electromagnetic waves. The resonance results from the excitation of high- $Q$ antisymmetric trapped current mode and shows sharp phase dispersion characteristic to Fano-type resonances of the electromagnetically induced transparency phenomenon. (C) 2009 American Institute of Physics. [DOI: 10.1063/1.3138868]
\end{abstract}

Planar metamaterials or metafilms are very thin metal films that are patterned on a subwavelength scale. Initially used as microwave frequency selective surfaces (FSS), 1,2 metafilms now attract attention due to their unusual functionalities ranging from invisibility, ${ }^{3}$ magnetic mirror ${ }^{4}$ and optical magnetism ${ }^{5}$ to asymmetric transmission ${ }^{6}$ and optical activity, $^{7}$ and have become a subject of intense research in the terahertz domain demonstrating application potential in wave manipulation and sensing. ${ }^{8-11}$ The response of metafilms can also be engineered to mimic electromagnetically induced transparency (EIT) of atomic systems. ${ }^{12-17}$ The spectral response of most FSSs, however, depends strongly on the polarization and, more importantly, on the angle of incidence of input waves. Limited attempts were made to reduce their angular sensitivity, ${ }^{18-22}$ which led to a more complex structures exploiting bilayered compositions ${ }^{23}$ and, more recently, fractal ${ }^{24,25}$ and volume split-ring resonators. ${ }^{26}$

In this letter we investigate a planar metamaterial, which exhibits characteristic EIT-like response with resonant frequency that is practically independent of the polarization and angle of incidence, and thus offers intriguing opportunities for developing optical delay, "slow light" and spectral filtering devices. Moreover, the metamaterial structure has a simple design ideally suited for the existing manufacturing planar micro- and nanotechnologies.

Our metamaterial is formed by a planar regular array of pairs of concentric subwavelength ring resonators (as shown in Fig. 1), which have recently been considered for achieving negative refraction in bulk composites. ${ }^{27,28}$ The double-ring (DR) pattern was etched from $35 \mu \mathrm{m}$ copper cladding covering FR4 PCB substrate of $1.6 \mathrm{~mm}$ thickness. The unit cell of the pattern contains one DR and is a square having size of $15 \times 15 \mathrm{~mm}^{2}$ (see Fig. 1). Such a metamaterial grating does not diffract at frequencies below $10 \mathrm{GHz}$ for any angle of incidence. The overall size of the sample was approximately $220 \times 220 \mathrm{~mm}^{2}$, which enabled us to conduct transmission measurements for incidence angles ranging from $0^{\circ}$ to $70^{\circ}$. Our measurements were performed in a microwave anechoic chamber in the 4-10 GHz frequency range using linearly polarized broadband horn antennas (Schwarzbeck BBHA

\footnotetext{
${ }^{a)}$ Electronic mail: vaf@orc.soton.ac.uk.

${ }^{b)}$ URL: www.nanophotonics.org.uk/niz.
}

9120D) equipped with dielectric lens concentrators and a vector network analyzer (Agilent E8364B).

Figure 2 shows a typical response of the planar metamaterial structure to normally incident linearly polarized waves, which was recorded in both transmission and reflection modes. It should be noted that although the data are presented for the polarization parallel to the structure's $y$-axis (as defined in Fig. 1), the response of the metamaterial showed no polarization dependence, which is consistent with the symmetry of its unit cell. The measured spectra reveal a very wide transmission stop-band split by a sharp asymmetric resonant feature at around $6.2 \mathrm{GHz}$ (marked as II in Fig. 2 ). In reflection it is seen as a narrow dip, where the reflectivity level drops below 5\%, separating two broad reflection resonances centered at correspondingly $5.8 \mathrm{GHz}$ (I) and 8.3 $\mathrm{GHz}$ (III). In transmission it corresponds to a narrow window with the transmission level exceeding $65 \%$ and the spectral width of about $0.7 \mathrm{GHz}$ measured as a full width at half maximum $(Q \approx 9)$. Moreover, the transmission window is accompanied by a very sharp normal phase dispersion [see Fig. 2(b)] that, as was recently pointed out in Refs. 13 and 14 , renders the response of the structure a metamaterial ana$\log$ of EIT and despite the structure's vanishing thickness can potentially be used for achieving long pulse delays. Importantly, the response of a reference metamaterial composed of the rings of large radius shows no splitting of the stop-band, which has $Q \approx 1$.

The origin of the narrow resonance can be traced to the excitation of the so-called trapped mode, a high- $Q$ mode formed by counter-propagating currents. Such a mode is nor-

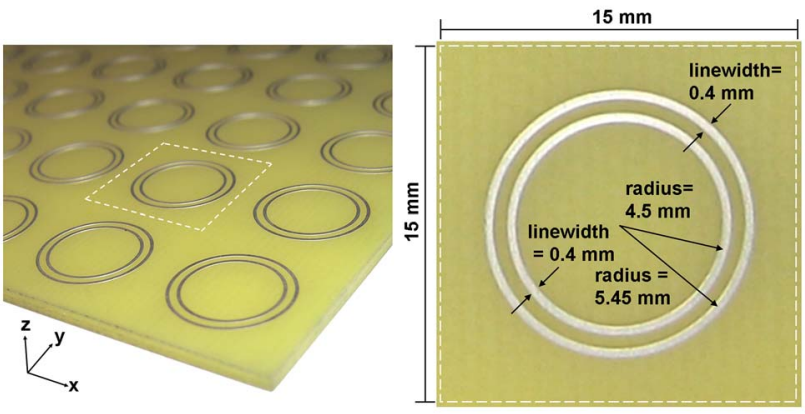

FIG. 1. (Color online) Photograph of the actual metamaterial structure Dashed box indicates its elementary unit cell. 

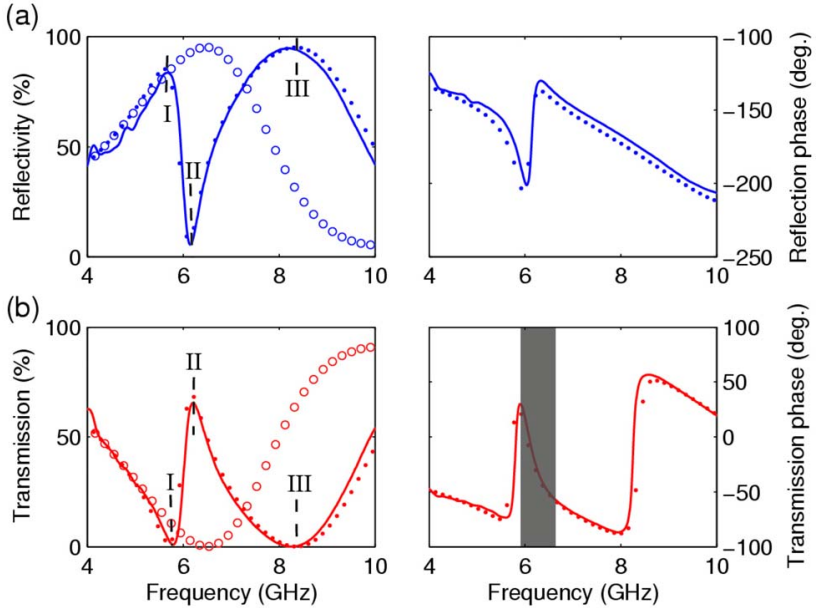

FIG. 2. (Color online) Normal incidence reflection (a) and transmission (b) spectra of the planar metamaterial with corresponding phase dispersions: solid line-experimental data, filled circles - theory (method of moments), empty circles-modeled reference structure composed of single large rings. Gray box indicates area of sharp normal phase dispersion.

mally inaccessible but can be excited if, for example, the metamaterial's structural elements have a certain weak structural asymmetry. ${ }^{12}$ As a result of the interference with the continuum-like spectrum of the excitation the mode acquires an asymmetrically shaped resonance line characteristic to the well-known Fano resonances. ${ }^{29}$

The trapped-mode nature of the observed pass-band was confirmed by numerical simulations, which showed exceptionally good agreement with the experimental results for both transmission and reflection, assuming $\epsilon=4.5-i 0.1$ (see Fig. 2, filled circles). In Fig. 3 we present the modeled distribution of currents in the metamaterial's unit cell at frequencies corresponding to the resonant features I, II, and III. Figures 3(a) and 3(c) show that the stop-bands I and III are the result of resonant excitation of a predominantly dipole mode (i.e., current oscillations symmetric with respect to $y$-axis) in either outer or inner ring, when the wavelength of excitation is approximately equal to the circumference of the corresponding ring. At the transmission resonance II, however, both rings appear to be excited equally, while the in- (a)
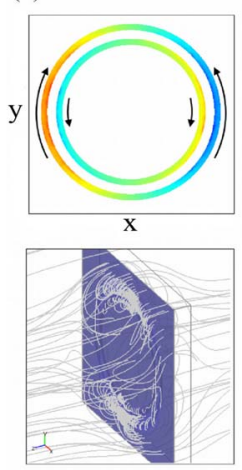

(b)

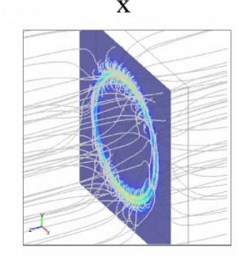

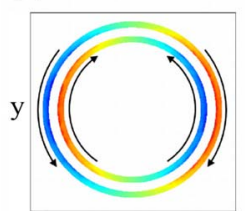

(c)

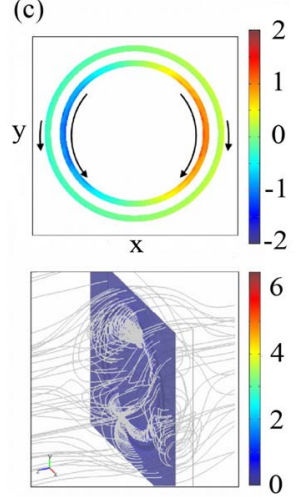

FIG. 3. (Color online) Modeling resonant excitations in the metamaterial unit cell. Panels (a)-(c) correspond to frequencies of 5.8,6.2, and $8.3 \mathrm{GHz}$, respectively (marked as I, II, and III in Fig. 2). Top row shows angular distribution of the surface current density $\left(j_{\phi}\right)$ in the rings, while the bottom row presents distribution of energy density and lines of power flow streaming through the structure. duced currents in the inner and outer rings oscillate in opposite phase yielding an electromagnetically trapped mode [see Fig. 3(b)]. Indeed, the scattered fields produced by such current configurations are very weak since the electrical dipole moment of each unit is negligible, while magnetic dipole and higher multipole moments such as, for example, electric quadrupole are absent due to the symmetry of the mode. As a consequence, the coupling of the metamaterial grating to free space and therefore its radiation losses are reduced dramatically, which ensures much stronger currents and thus higher $Q$-factor of the response than in the case of dipole excitation.

The difference between the two regimes of excitation is further illustrated in Fig. 3 in terms of calculated power flow, as well as local distributions of energy density in the plane of the rings. Remarkably, at the trapped-mode resonance the flow of electromagnetic energy is confined to a very small region between the rings, where the energy density reaches substantially high values when compared to the case of dipole excitation. This makes the response of the metamaterial operating in the trapped-mode regime extremely sensitive to the dielectric properties of the substrate (or a superstrate), which may potentially be used for enhancing optical nonlinearities or efficient single-pass amplification of light in the nanoscaled versions of the metamaterial. ${ }^{30}$

It is important to note that in the DR-structure excitation and control of the trapped-mode resonance requires no symmetry breaking, therefore the structure's electromagnetic properties are different from those of the recently demonstrated asymmetrically split rings. ${ }^{12}$ In the present case the free-space coupling of DRs (and consequently the width of the narrow transmission window) is exclusively controlled by the difference in circumferences of the inner and outer rings and does not depend on the polarization state of the incident electromagnetic radiation. Importantly, due to vanishing electric dipole moment and absence of magnetic dipole moment in the DRs at the trapped-mode resonance, their mutual interactions are extremely weak. This makes our planar metamaterial a truly incoherent system, where the response of the entire array is simply a direct sum of the DR individual contributions. ${ }^{31}$ As a consequence the electromagnetic response of the metamaterial is also weakly dependent on the angle of incidence, i.e., appear isotropic for a wide range of angles. Such remarkable behavior is illustrated in Fig. 4, where we present metamaterial transmission measured as a function of frequency and angle of incidence. It is evident that at normal incidence the central frequency $(\approx 6.2 \mathrm{GHz})$ and width $(\approx 0.7 \mathrm{GHz})$ of the transmission band are the same for both TE and TM polarizations and remains fairly stable for a wide range of angles. In particular, the peak amplitude and width change by only about $\pm 10 \%$ and $\pm 25 \%$, respectively, for angles of up to $45^{\circ}$, while the band's central frequency remains the same within experimental accuracy for the whole range of angles measured. This renders the resonant response of the metamaterial structure polarization and angle independent for most of applications. We anticipate that by reducing the gap between the concentric rings the strength of their resonant free-space coupling can be reduced further, which will result in the narrower transmission line and simultaneously extend the range of acceptable angles beyond $45^{\circ}$. It is worth mentioning that a narrow stop-band response with similar unique character- 


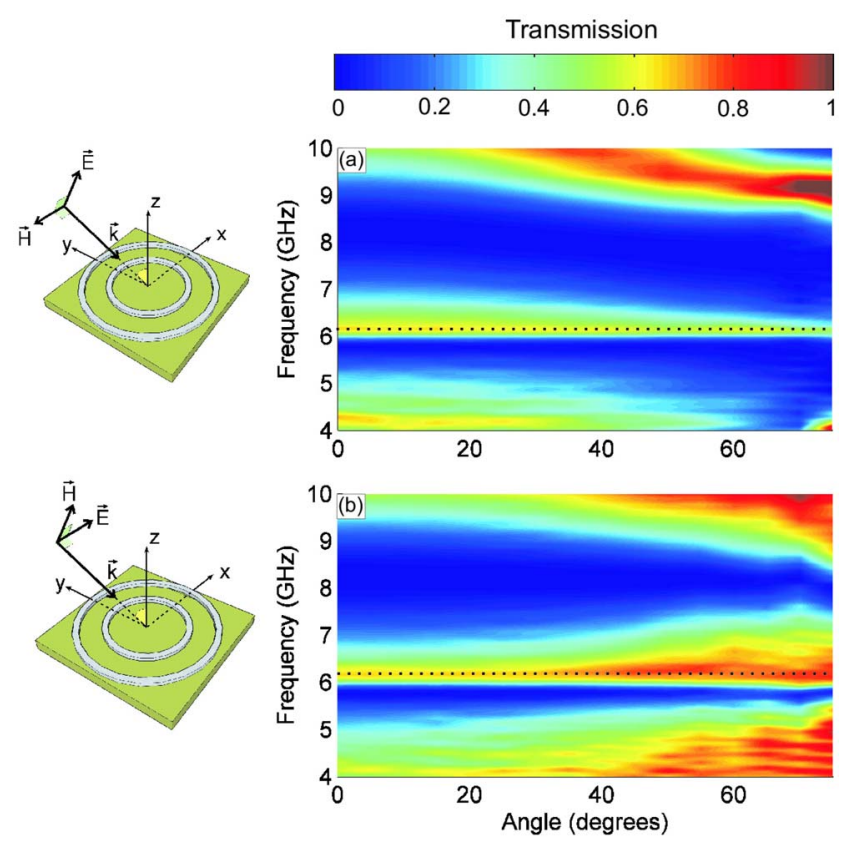

FIG. 4. (Color online) Angular dependence of the metamaterial's transmission spectrum measured for (a) TM and (b) TE incident polarizations. Dashed lines indicate spectral location of the trapped-mode resonance. Insets show mutual orientations of the electric and magnetic vectors of the incident wave and the metamaterial's unit cell at oblique incidence.

istics can be obtained by simply using a complementary DRstructure, where metal rings are replaced with concentric ring apertures in a metal film.

In summary, we designed and experimentally demonstrated a planar metamaterial (metafilm) exhibiting polarization and angle independent narrow pass-band, which shall have potential applications in wide-acceptance-angle detectors. Such unusual for metafilms isotropy of the spectral response is a result of engaging trapped-mode resonance in the absence of structural symmetry breaking. The EIT-like phase dispersion associated with the resonance makes our metamaterial also an intriguing candidate for the use in "slow light" applications, while strong spatial localization of energy provided by the trapped mode can potentially be exploited for enhancing optical nonlinearities or efficient single-pass amplification of light in micro- and nanoscaled versions of the metamaterial, which are fully compatible with the existing fabrication planar technologies.

This work has been supported by the EPSRC (UK) via the Nanophotonics Portfolio grant, International Collaborative grant with National Taiwan University and a CA Fellowship (V.A.F.), and National Academy of Sciences of Ukraine (Grant No. 1-02-a).
${ }^{1}$ J. Vardaxoglou, Frequency Selective Surfaces (Research Studies, England, 1997).

${ }^{2}$ B. A. Munk, Frequency Selective Surfaces: Theory and Design (Wiley, New York, 2000).

${ }^{3}$ V. A. Fedotov, P. L. Mladyonov, S. L. Prosvirnin, and N. I. Zheludev, Phys. Rev. E 72, 056613 (2005).

${ }^{4}$ D. Sievenpiper, L. Zhang, R. Broas, N. Alexopolous, and E. Yablonovitch, IEEE Trans. Microwave Theory Tech. 47, 2059 (1999).

${ }^{5}$ S. Linden, C. Enkrich, G. Dolling, M. W. Klein, J. Zhou, T. Koschny, C. M. Soukoulis, S. Burger, F. Schmidt, and M. Wegener, IEEE J. Sel. Top. Quantum Electron. 12, 1097 (2006).

${ }^{6}$ V. A. Fedotov, P. L. Mladyonov, S. L. Prosvirnin, A. V. Rogacheva, Y. Chen, and N. I. Zheludev, Phys. Rev. Lett. 97, 167401 (2006).

${ }^{7}$ E. Plum, V. A. Fedotov, and N. I. Zheludev, Appl. Phys. Lett. 93, 191911 (2008).

${ }^{8}$ H.-T. Chen, W. J. Padilla, J. M. O. Zide, A. C. Gossard, A. J. Taylor, and R. D. Averitt, Nature (London) 444, 597 (2006).

${ }^{9}$ R. Singh, E. Smirnova, A. J. Taylor, J. F. O'Hara, and W. Zhang, Opt. Express 16, 6537 (2008).

${ }^{10}$ R. Singh, A. K. Azad, J. F. O’Hara, A. J. Taylor, and W. Zhang, Opt. Lett. 33, 1506 (2008).

${ }^{11}$ J. F. O'Hara, R. Singh, I. Brener, E. Smirnova, J. Han, A. J. Taylor, and W. Zhang, Opt. Express 16, 1786 (2008).

${ }^{12}$ V. A. Fedotov, M. Rose, S. L. Prosvirnin, N. Papasimakis, and N. I. Zheludev, Phys. Rev. Lett. 99, 147401 (2007).

${ }^{13}$ N. Papasimakis, V. A. Fedotov, N. I. Zheludev, and S. L. Prosvirnin, Phys. Rev. Lett. 101, 253903 (2008).

${ }^{14}$ S. Zhang, D. A. Genov, Y. Wang, M. Liu, and X. Zhang, Phys. Rev. Lett. 101, 047401 (2008).

${ }^{15}$ N. Liu, S. Kaiser, and H. Giessen, Adv. Mater. (Weinheim, Ger.) 20, 4521 (2008).

${ }^{16}$ P. Tassin, L. Zhang, T. Koschny, E. N. Economou, and C. M. Soukoulis, Phys. Rev. Lett. 102, 053901 (2009).

${ }^{17}$ R. Singh, C. Rockstuhl, F. Lederer, and W. Zhang, Phys. Rev. B 79, 085111 (2009).

${ }^{18}$ A. Roberts and R. C. McPhedran, IEEE Trans. Antennas Propag. 36, 607 (1988).

${ }^{19}$ J. Shaker and L. Shafai, Electron. Lett. 29, 1655 (1993).

${ }^{20}$ J. Huang, T.-K. Wu, and S.-W. Lee, IEEE Trans. Antennas Propag. 42, 166 (1994).

${ }^{21}$ T.-K. Wu and S.-W. Lee, IEEE Trans. Antennas Propag. 42, 1484 (1994).

${ }^{22}$ D. Van Labeke, D. Gerard, B. Guizal, F. I. Baida, and L. Li, Opt. Express 14, 11945 (2006).

${ }^{23}$ J. Shaker and L. Shafai, IEEE Microw. Guid. Wave Lett. 5, 324 (1995).

${ }^{24}$ L. Zhou, C. T. Chan, and P. Sheng, J. Phys. D 37, 368 (2004).

${ }^{25}$ W. Wen, L. Zhou, B. Hou, C. T. Chan, and P. Sheng, Phys. Rev. B 72, 153406 (2005).

${ }^{26}$ J. D. Baena, L. Jelinek, R. Marques, J. J. Mock, J. Gollub, and D. R. Smith, Appl. Phys. Lett. 91, 191105 (2007).

${ }^{27}$ Z.-G. Dong, M.-X. Xu, S.-Y. Lei, H. Liu, T. Li, F.-M. Wang, and S.-N. Zhu, Appl. Phys. Lett. 92, 064101 (2008).

${ }^{28}$ P. Ding, E. J. Liang, L. Zhang, Q. Zhou, and Y. X. Yuan, Phys. Rev. E 79, 016604 (2009).

${ }^{29}$ U. Fano, Phys. Rev. 124, 1866 (1961).

${ }^{30}$ N. I. Zheludev, S. L. Prosvirnin, N. Papasimakis, and V. A. Fedotov, Nat. Photonics 2, 351 (2008).

${ }^{31}$ N. Papasimakis, V. A. Fedotov, Y. H. Fu, D. P. Tsai, and N. I. Zheludev, arXiv:0809.2361. 